\title{
An API/GUI for Monte Carlo Simulation of EPMA Spectra Using PENELOPE
}

\author{
Philippe T. Pinard*, Hendrix Demers**, Francesc Salvat***, Raynald Gauvin*
}

* Materials and Mining Engineering. Department., McGill University, 3610 University St., Montréal, Québec, Canada H3A 2B2

** Département de génie électrique et génie informatique, Université de Sherbrooke, 2500 boul. Université, Sherbrooke, Québec Canada J1K 2R1

*** Facultat de Fisica (ECM), Universitat de Barcelona, Diagonal 647, Barcelona, Spain 08028

PENELOPE [1] is a Fortran Monte Carlo code for simulation of coupled electron-photon transport matter that allows a detailed interpretation of experimental results of electron spectroscopy and microscopy. PENEPMA [2] is a dedicated main program of PENELOPE designed to simulate electron microanalysis experiments with $\mathrm{x}$-ray detectors. PENELOPE implements an elaborate algorithm for electron transport that uses accurate cross-sections for elastic scattering and accounts for energy-straggling effects. Photon interactions are simulated in chronological succession, allowing the calculation of x-ray fluorescence in complex geometries. The drawback is significantly longer simulation time than for simpler Monte Carlo codes. The aim of the present work is twofold: the optimization of the PENELOPE simulation parameters to reduce the simulation time of $x$-ray spectra, and the development of an application programming (API) and graphical user interface (GUI) to facilitate the use of PENEPMA.

The PENELOPE simulation parameters were optimized to obtain the maximum efficiency for a variety of experimental conditions without any appreciable loss of accuracy. In the optimization study, a subset of thirteen cases was selected from the Pouchou and Pichoir [3] k-ratios database of binary alloys. These cases were chosen to reflect good and bad agreement between simulation results and experimental data, and we included also problematic/representative cases with a large absorption or fluorescence contribution. The effect of the simulation parameters on the efficiency, the backscattered coefficient and the intensity of the X-ray line of interest was studied. For the scattering parameters $C 1$ and $C 2$ (which determine the mean free path between hard elastic events), the results show that using values of about 0.02 procures large efficiency improvements (Figure 1) with no significant changes in the backscattered coefficients and X-ray intensities. PENEPMA makes extensive use of interaction forcing (a variance-reduction technique which artificially increases the probability of occurrence of relevant interactions) to improve the efficiency. By simultaneously forcing the bremsstrahlung emission and inner-shell ionization, up to 8-fold efficiency gain was obtained without compromising the accuracy of the calculated x-ray intensities (Figure 2). However, it was found that forcing Compton scattering and photoelectric absorption decreases the global efficiency except for one high fluorescence case. Forcing of photon interactions does improve the accuracy of fluorescent contributions, but slows down the global simulation speed.

Written in Python, the developed API allows the user to create a large series of PENEPMA simulations using object-oriented programming and to interpret efficiently the results from these simulations. These functionalities are also coupled with a graphical interface which implements the optimized simulation parameters indicated above, thus facilitating the setup of typical microanalysis simulations. The GUI removes the tedious steps of setting up the materials, geometry, simulation parameters and position of the detectors. New features are also added to the PENEPMA code to simulate electron showers and $\varphi(\rho z)$ curves as well as new stopping conditions. 
[1] F. Salvat, J.M. Fernández-Varea and J. Sempau, PENELOPE. A Code System for Monte Carlo Simulation of Electron and Photon Transport, (OECD/NEA Data Bank, Issy-les-Moulineaux, France, 2006).

[2] X. Llovet and F. Salvat, PENEPMA, a Monte Carlo code for the simulation of $x$-ray emission spectra using PENELOPE, 2008.

[3] J.L. Pouchou and F. Pichoir, Quantitative Analysis of Homogeneous or Stratified Microvolumes Applying the Model PAP, in: Electron Probe Quantitation, Plenum Press, New York, 1991.

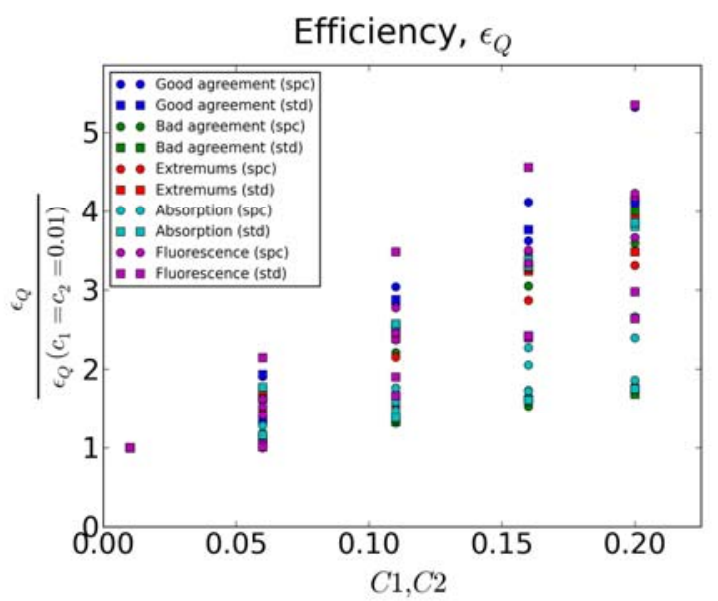

(a)

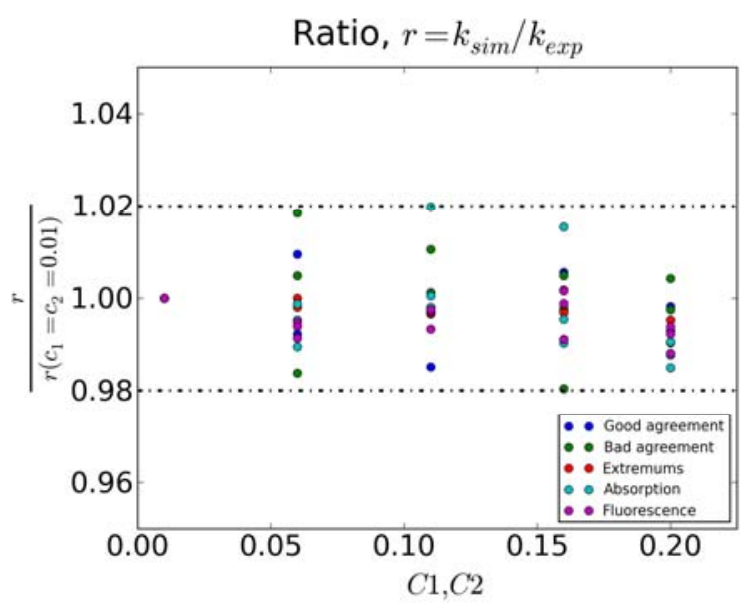

(b)

Fig. 1: Effect of the elastic-scattering parameters $\mathrm{C} 1$ and $\mathrm{C} 2$ on the efficiency (a) and on the x-ray intensity (b) of the 13 studied cases. All simulations were run until the error on the intensity of the $\mathrm{x}$-ray line of interest reached $1 \%$. This gives a $1.41 \%$ statistical error on the k-ratios calculations of Fig. $1 \mathrm{~b}$ (represented by dashed lines).

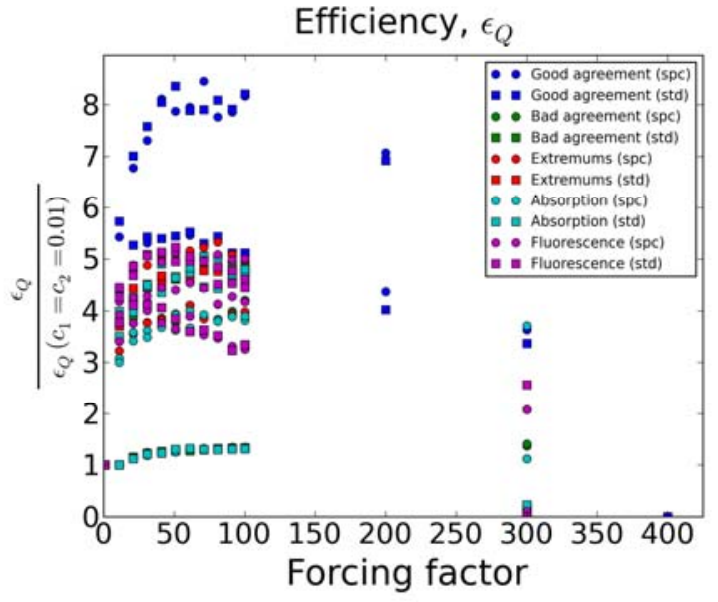

(a)

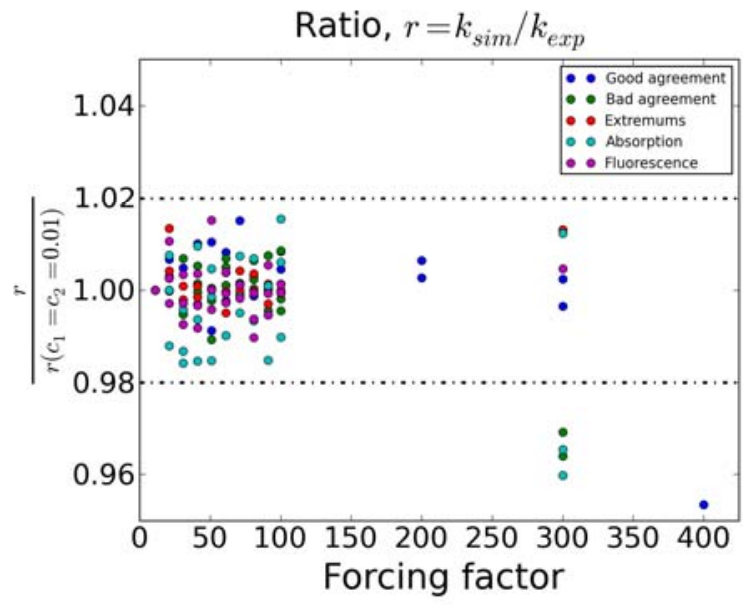

(b)

Fig. 2: Effect of forcing bremsstrahlung emission and inner-shell ionization on the efficiency (a) and on the X-ray intensity (b) of the 13 studied cases. Same simulation conditions as in Fig. 1. 